Sädhanä, Vol. 19, Part 5, October 1994, pp. 649-670. (C) Printed in India.

\title{
Flow transitions and pattern selection of the Rayleigh-Bénard problem in rectaugular enclosures
}

\author{
D MUKUTMONI ${ }^{1}$ and $\mathrm{K} T \mathrm{YANG}^{2}$ \\ ${ }^{1}$ Adapco Ltd, Melville, NY 11747, USA \\ ${ }^{2}$ Department of Aerospace and Mechanical Engineering, University of \\ Notre Dame, Notre Dame, IN 46556, USA
}

\begin{abstract}
In this paper, research efforts in the broad area of flow transitions of Rayleigh-Bénard convection in rectangular enclosures with sidewalls are reviewed. Numerical studies are given primary emphasis. However, experimental works that are relevant are described. Our current physical understanding of the transition phenomena as occurring in the RayleighBénard problem is critically reviewed. Two broad categories of transition are discussed. In the former, the transitions are temporal in nature, and mostly confined to small enclosures. In the latter, transitions are a result of change in spatial patterns. This phenomenon, known as pattern selection, is looked into for both small and intermediate enclosures.
\end{abstract}

Keywords. Rayleigh-Bénard problem; rectangular enclosure; flow transition; pattern selection.

\section{Introduction}

Rayleigh-Bénard convection is among the most heavily investigated physical problems for nearly a century. The enduring popularity of the problem with respect to a wide cross section of scientists and engineers is not surprising. The RayleighBénard problem is relevant to applications ranging from astrophysics, geophysics, atmospheric sciences and various disciplines of engineering. Many familiar and esoteric physical phenomena such as the imperceptible movement of the continental plates, the violent magnetic storms in the solar atmosphere, the destructive forces of a tropical cyclone are in essence a manifestation of the Rayleigh-Bénard convection for different geometries and parametric ranges. In an industrial context, RayleighBénard (henceforth abbreviated to RB at times) convection has applications in disciplines such as solar energy systems, energy storage, material processing and nuclear reactor systems.

RB convection forms a subclass of fluid flow problems that are known as buoyancydriven flows or thermal convection. In these, fluid flow is induced by density differences that arise as a result of temperature differences. Thermal convection occurring in enclosures or cavities caused by a temperature gradient in the direction of the gravity vector is known as Rayleigh-Bénard convection. Other than its relevance to the 
various disciplines of the physical sciences, the RB problem has been investigated for theoretical and fundamental reasons as well. The problem is governed by nonlinear coupled partial differential equations. It therefore serves as a paradigm of a nonlinear system. The model problem has been used to investigate the transition from laminar to turbulent flow. In another related issue, the physical model has been used to study the nonlinear selection and evolution of patterns. These two aspects of RB convection will be the subject of this paper.

More specifically, we review and highlight some of the computational work that has been done to study the phenomena of flow transition and pattern formation with respect to Rayleigh-Bénard convection. In the process, we will also include some of the relevant experimental work. This is important, since the only true test of the correctness of the computational model is reasonable agreement with experiments. The twin areas offer an exciting area of research with far-reaching implications.

\section{Brief background of the Rayleigh-Benard problem}

The Rayleigh-Bénard problem in its simplest form and one that was the earliest to be investigated is the so-called infinite layer case. In such a case, a layer of fluid is constrained between two infinite horizontal surfaces. The system is heated from below, i.e., the lower surface is at a higher temperature than the upper surface. The heatedfrom-below case is said to have an adverse temperature gradient because the fluid at the bottom will be lighter than the fluid at the top and this top-heavy arrangement is potentially unstable. When the temperature gradient is below a certain value, the natural tendency of the fluid to move, because of buoyancy, will be inhibited by its own viscosity and thermal diffusivity. Thus, the thermal instability will manifest itself only when the adverse temperature gradient exceeds a certain critical value.

The earliest experiments to demonstrate in a definitive manner the onset of thermal instability in fluids are due to Bénard (1900). The theoretical foundations for a correct interpretation of the phenomena are due to Rayleigh (1916). The phenomenon of thermal convection under an adverse temperature gradient is therefore known as the Rayleigh-Bénard convection in their honour. The non-dimensional adverse temperature gradient is known as the Rayleigh number. An introductory exposition and review of the infinite layer RB convection can be found in Chandrasekhar (1961). A more advanced and complete review of the infinite layer RB convection instability at higher Rayleigh numbers is given in Busse (1978). For a general discussion of transitions and bifurcations for buoyancy-driven enclosure flows, the reader is referred to Yang (1988).

Although it will be of immense benefit to the research community to review all current work done with regard to flow transitions in natural convection, it is felt that the scope is too vast to be included in just a single article. We therefore chose a more specialized topic and restricted ourselves to the numerical study of flow transitions, instabilities and bifurcations for the Rayleigh-Bénard problem in rectangular enclosures with sidewalls only. In the following sections, the basic RB problem is formulated and the various physical parameters discussed. This is followed by a general discussion of RB convection with respect to flow transitions primarily restricted to small enclosures and temporal transitions. Following that, we discuss RB convection restricted to spatial transitions in small and intermediate boxes. All along we emphasize the 
numerical methods used by the researchers in their study and relevant experimental works. We conclude with a recommendation on future study.

\section{Problem statement}

The problem formulation described in this section was for most part used for most of the numerical work reviewed and in particular to all our recent investigations. RB convection is studied in a three-dimensional rectangular geometry. The geometry of the enclosure is shown in figure 1 . The vertical walls are adiabatic. The bottom wall is heated and the top wall is cooled, both isothermally. The Boussinesq approximation is invoked. Consequently, all transport properties are assumed constant with the exception of the buoyancy term in the momentum equations, which is linearized. The governing equations are non-dimensionalized by suitable scales of the dependent and independent variables. The $x, y$ and $z$ coordinates are non-dimensionalized by $L$, the enclosure height. The velocities, time and pressure were scaled by $\alpha / L, L^{2} / \alpha$ and $\rho L^{2} / \alpha^{2}$ respectively. In this case, $\alpha$ is the thermal diffusivity of the fluid. The temperature was normalized with respect to the top and bottom wall temperatures. The non-dimensionalized governing equations are the continuity, the Navier-Stokes and the energy equations that are listed below:

$$
\begin{aligned}
& \nabla \bullet \mathbf{U}=0, \\
& (\partial u / \partial t)+\nabla \bullet(u \mathbf{U})=-(\partial p / \partial x)+\operatorname{Pr} \nabla^{2} u, \\
& (\partial v / \partial t)+\nabla \bullet(v \mathbf{U})=-(\partial p / \partial y)+\operatorname{Pr} \nabla^{2} v+\operatorname{RaPr} T, \\
& (\partial w / \partial t)+\nabla \bullet(w \mathbf{U})=-(\partial p / \partial z)+\operatorname{Pr} \nabla^{2} w, \\
& (\partial T / \partial t)+\nabla \bullet(T \mathbf{U})=\nabla^{2} T .
\end{aligned}
$$

The boundary conditions that are consistent with the adiabatic and isothermal walls

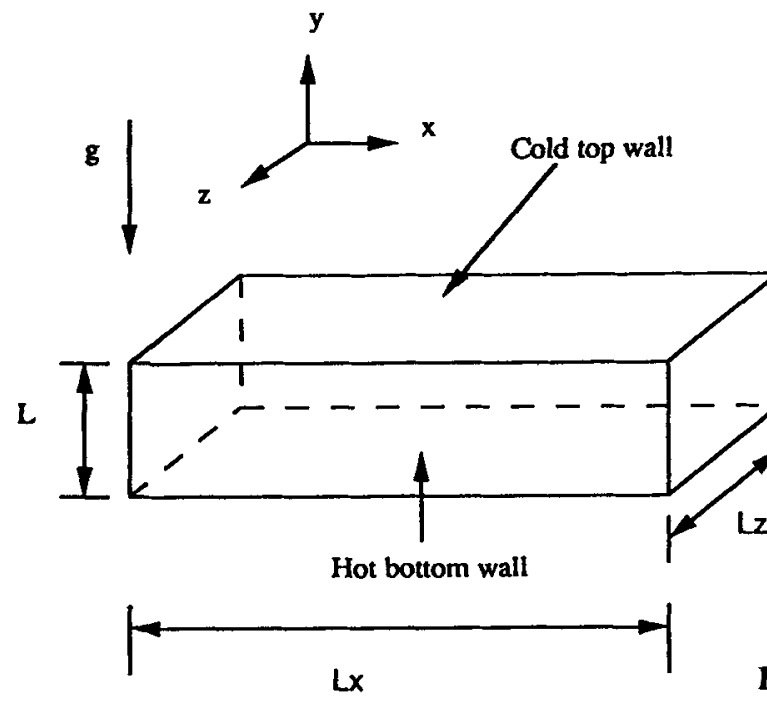

Figure 1. Geometry of the enclosure. 
in a non-dimensional form are the following:

$$
\begin{aligned}
& x=0, A_{x} ; 0 \leqslant z \leqslant A_{z} ; 0 \leqslant y \leqslant 1 ; u=v=w=0(\partial T / \partial x)=0 \\
& z=0, A_{z} ; 0 \leqslant x \leqslant A_{x} ; 0 \leqslant y \leqslant 1 ; u=v=w=0(\partial T / \partial z)=0 \\
& y=0,1 ; 0 \leqslant x \leqslant A_{x} ; 0 \leqslant z \leqslant A_{z} ; u=v=w=0 T=0 \cdot 5-y
\end{aligned}
$$

The significant parameters of the formulation are the Rayleigh number $\mathrm{Ra}$ that represents the strength of the driving forces and the Prandtl number Pr. They are defined as follows;

$$
\mathrm{Ra}=g \beta \Delta T L^{3} /(v \alpha) ; \quad \operatorname{Pr}=v / \alpha .
$$

The two other parameters are the normalized dimensions of the rectangular enclosure in the horizontal directions or the geometrical aspect ratios $A_{x}$ and $A_{z}$. For problems in which some of the assumptions are relaxed, there will be additional parameters. For instance if the Boussinesq approximation is not used, a parameter to quantify the departure from the Boussinesq approximation will arise. These additional parameters will be discussed on a case-by-case basis wherever applicable.

\section{Bifurcation to convective flow from initial conduction state}

It has generally been known that for the given problem, as the Rayleigh number is increased, the system undergoes a series of bifurcations. By a bifurcation we mean that the flow undergoes a qualitative change in the flow and temperature field. For instance, an increase in the Rayleigh number and a corresponding decrease in the number of rolls would exemplify a flow bifurcation. On the other hand, if an increase in the Rayleigh number merely increases the velocities and heat transport without changing the flow pattern, no bifurcation would have occurred.

Below a certain critical Rayleigh number, there is no motion since the buoyant forces cannot overcome the viscous forces. Davis (1967) and Catton (1970) calculated the critical Rayleigh number for the onset of convection by a linear stability analysis of the motionless conduction state. The eigenvalue problem was solved by a Galerkin procedure wherein the dependent variables are expressed as a summation of trial functions as shown below,

$$
\mathrm{U}=\sum_{n=1}^{N} c_{n} \varphi_{n}, T=\sum_{n=1}^{N} d_{n} \psi_{n}, p=\sum_{n=1}^{N} e_{n} \zeta_{n} .
$$

Similar calculations by Bühler et al (1979) using a more complete set of trial functions showed that the critical Rayleigh number is a function of the geometrical aspect ratios. The critical Rayleigh number for the onset of motion decreases with an increase in the aspect ratio and is the lowest for the infinite layer case which is 1707.8 . The critical Rayleigh numbers for cylindrical lateral walls was calculated by Charleson $\&$ Sani (1970).

\section{Transition to time dependence}

When the Rayleigh number is increased beyond a certain critical value, RB convection becomes oscillatory through an instability mechanism predicted for the two-dimensional 
infinite layer case by Clever \& Busse $(1974,1987)$ using linear stability theory. Stability analysis beyond oscillatory instability has not been reported. However, experimental results of large aspect ratio enclosure flow by Ahlers \& Behringer (1978) indicate that the flow becomes turbulent soon after oscillatory convection. For intermediate aspect ratio containers (aspect ratios between 5 and 30), experiments by Oertel (1982, pp. 3-24) indicate a spatially complex structure. Walden et al (1984) discovered from their measurements that the temporal behaviour of the fluid was spatially dependent.

In contrast, $\mathrm{RB}$ convection in small aspect ratio as reported in experiments (Maurer \& Libchaber 1979; Gollub \& Benson 1980; Bergé et al 1982, pp. 123-148) indicate that the dynamical behaviour of thermal convection follows a well-defined set of bifurcations to chaos and turbulence as the Rayleigh number is increased. The dynamical behaviour is simpler due to the restricting influence of the side-walls. A more interesting general observation was that the bifurcation sequences were qualitatively similar to simple dynamical systems such as nonlinear ordinary difference and differential equations. Examples of bifurcation sequences or scenarios include the Ruelle-Takens scenario (Ruelle \& Takens 1971) and the Feigenbaum sequence (Feigenbaum 1978). For a more complete documentation of bifurcation in simple dynamical systems, the reader is referred to Parker \& Chua (1988).

A brief description of the experiments is in order. In the experiments of Gollub \& Benson (1980), two enclosures $3.5 \times 2.1 \times 1$, and $2.4 \times 1.2 \times 1$, and two different Prandtl numbers ( 2.5 and 5 ) were investigated. Four major transition sequences to turbulent flows were reported. One significant result was that the transition depended not only on the Prandtl number and geometry but on the initial conditions as well. The number of different flow configurations was surprisingly many for a small aspect ratio box.

Experiments on a small box $(2 \times 1.2 \times 1)$ with silicone oil $(\operatorname{Pr}=130)$ were carried out by Bergé et al (1982, pp. 123-48) and Arroyo \& Savirón (1992). The observed routes to chaos were similar to those observed for fluids with lower Prandtl numbers such as air (Kirchartz \& Oertel 1988). Maurer \& Libchaber (1979) performed experiments with liquid helium $(\mathrm{Pr}=0 \cdot 5)$ and observed similar routes to turbulence. Although the dynamical behaviours were not identical, one common feature noted was that the first bifurcation to time-dependent behaviour is oscillatory with a single independent frequency. Furthermore, the critical Rayleigh number for the onset of time-dependent flow decreased with a reduction of the fluid Prandtl number.

\section{Relevance of numerical studies}

The central problem confronting a fluid dynamicist is the phenomena of turbulence. Due to the unacceptably fine mesh required to resolve the length scales of turbulence, it is impossible to numerically simulate turbulence in flows that occur in practical applications, given present computing resources. One approach to understanding the phenomena of turbulence better would be to completely solve a simpler problem that nevertheless shares many of the features of turbulence. One such "simpler problem" is the Rayleigh-Bénard problem in small aspect ratio enclosures in the unsteady and chaotic regime. Nevertheless, computations in an RB system are not particularly easy.

Such computations must necessarily be three-dimensional using numerical schemes with low numerical diffusion and hence higher order and computationally expensive. 
Also, since the flow is time-dependent, the integration with respect to time must be carried out for long intervals. With the phenomenal increase in computing power in the past several years, these problems have become tractable and represent a very exciting area of research. By an accurate numerical simulation of well-documented bifurcation experiments, one could begin to understand the phenomena of bifurcation and transition better. Insights into the physics of the RB system could potentially lead to a better understanding to the important problem of transition to turbulent flows.

The study of bifurcation phenomena in small enclosures is also a fascinating problem in its own right. Busse and coworkers have more or less identified, in a complete manner, the instabilities associated with roll convection in infinite enclosures. The extension to finite boxes, especially small boxes, is not straightforward, and practically intractable from the point of view of a stability problem since the base state is analytically complex. The only tool available is a complete and accurate numerical simulation of the basic equations. The documentation of all instability mechanisms associated with small and intermediate enclosures represents a formidable and challenging enterprise for applied mathematicians, physicists and engineers.

\section{Early numerical study of the RB system}

Numerical investigation of the bifurcation phenomena in small boxes has been few and very recent. Upson et al (1981, pp. 245-59) used a modified Galerkin finite element method to simulate thermal convection for the case experimentally investigated by Maurer \& Libchaber (1979) for a $3.29 \times 1.8 \times 1$ enclosure and a Prandtl number of 0.5 . The frequencies of oscillations were close to that in the experiments. Two different flow patterns, one with two rolls and the other with three rolls were found to occur for the same boundary conditions (but different initial conditions). However, the transition from three to two rolls that was documented experimentally could not be reproduced. Mukutmoni \& Yang (1992) numerically simulated the transition from three to two rolls. It is possible that the simulations of Upson et al (1981, pp. 245-59) were not carried out long enough for the instability to manifest itself.

Kessler (1987) carried out simulations with a 4:2:1 box for air. Spectral Galerkin methods were used. The dependent variables were expanded in the form similar to (10),

$$
\psi=\sum_{i=1}^{L} \sum_{j=1}^{M} \sum_{k=1}^{N} a_{i j k}(t) C_{i}(x) C_{j}(y) C_{k}(z)
$$

The $C(x)$ 's are the so-called 'beam functions' that satisfy the zero normal and tangential velocity at the wall. As given in Chandrasekhar (1961), they have the following functional form:

$$
\begin{aligned}
C(x)=\left(\cosh \lambda_{m} x / \cosh \left(\lambda_{m} / 2\right)\right)-\left(\cos \lambda_{m} x / \cos \left(\lambda_{m} / 2\right)\right) \text { or } \\
\\
\left(\sinh \mu_{m} x / \sinh \left(\mu_{m} / 2\right)\right)-\left(\sin \mu_{m} x / \sin \left(\mu_{m} / 2\right)\right) .
\end{aligned}
$$

$\lambda_{m}$ and $\mu_{m}$ are the eigenvalues for a specific set of boundary conditions. For higher Rayleigh numbers where extra resolution is required for the boundary layers, Chebyshev polynomials were used. The numerical simulations looked into details of the steady and oscillatory convection. However, ad-hoc symmetry conditions were imposed to 
reduce computational costs. As a result, it was not a suitable method for studying flow transitions. In particular, the symmetry conditions forced an odd number of rolls.

Yahata (1984) computed for several aspect ratios including cylindrical side walls using a Galerkin method. The assumption of slip walls made the computations more efficient but less realistic. The calculations showed several possible routes to chaotic flow. Non-Boussinesq effects were investigated.

Urata (1986) used spectral methods to compute for a 2:2:1 box for Prandtl numbers of 1 and 3 . The study looked into the details of time-dependent convection. However, the assumption of slip-walls meant that the solution obtained was somewhat unrealistic. The computations spanned the chaotic regime. The Lyapunov exponents were calculated. For $\operatorname{Pr}=1$, and $\mathrm{Ra}=20,000$, the dimension of the chaotic attractor was determined to be $3 \cdot 3$.

\section{Some recent studies on small aspect ratio $\mathbf{R B}$ convection}

In this section we review some of the recent works on RB convection in small boxes. All studies reviewed in this section computed realistic cases (non-slip walls). More significantly, extensive comparisons with experiments were made. The RB system even for small aspect ratio enclosures permits multiple solutions to the numerical problem. Although some solutions computed without proper experimental validation are real, many of them are obtained from incorrect application of boundary conditions or inadequate resolution from a coarse mesh and are therefore not physical. If the purpose of the study is to understand the physics, comparison and validation from closely related experiments is imperative. As observed by Yang \& Mukutmoni (1992, pp. 23-41), only then can it be claimed that the numerical solution is indeed an actual solution.

Mukutmoni \& Yang (1993a) studied a two-roll RB convection for the case experimentally studied by Gollub et al $(1980$, pp. 22-7) for a $3 \cdot 5: 2 \cdot 1: 1$ box for a fluid of Prandtl number 2.5. The schematic diagram of the two-roll RB convection is shown in figure 2.

Due to the nonlinearity of the governing equations, the solution is not uniquely determined by the governing parameters. The solution depends on the initial conditions.

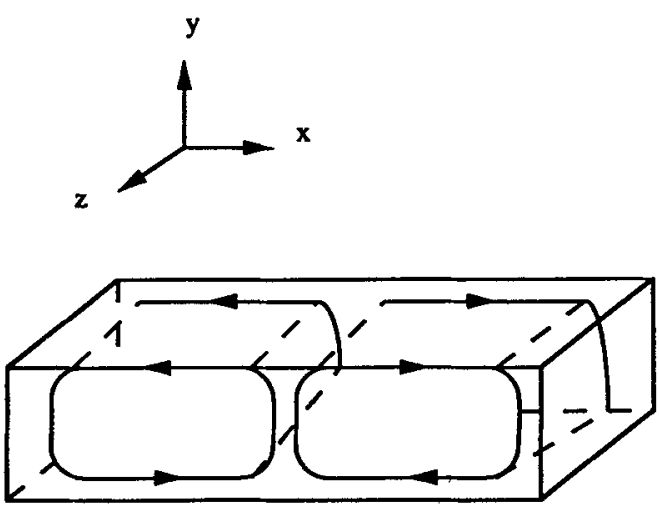

Figure 2. Schematic of a two-roll RB convection. 
(a)

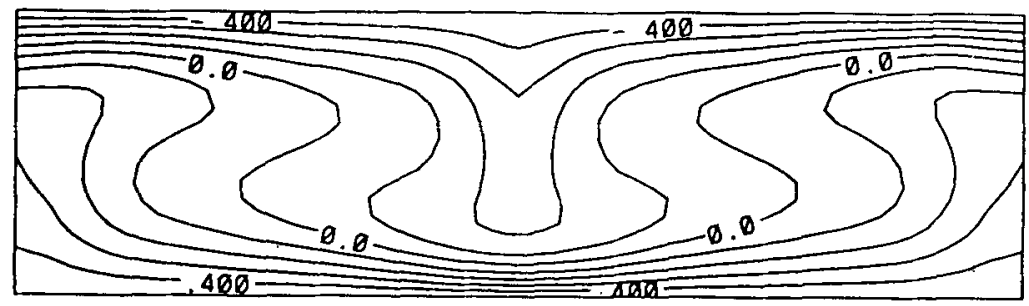

(b)

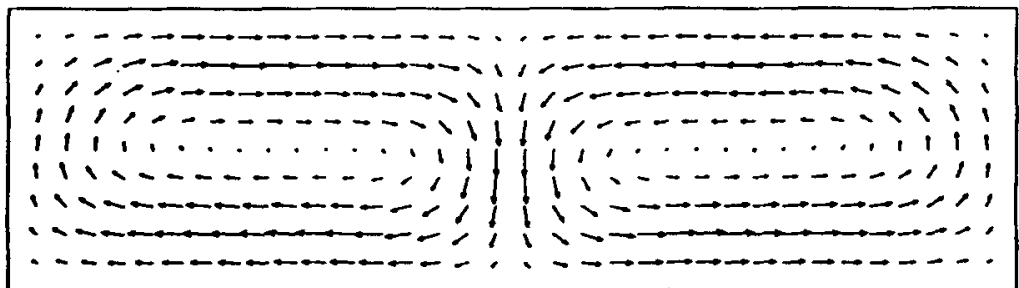

(c)

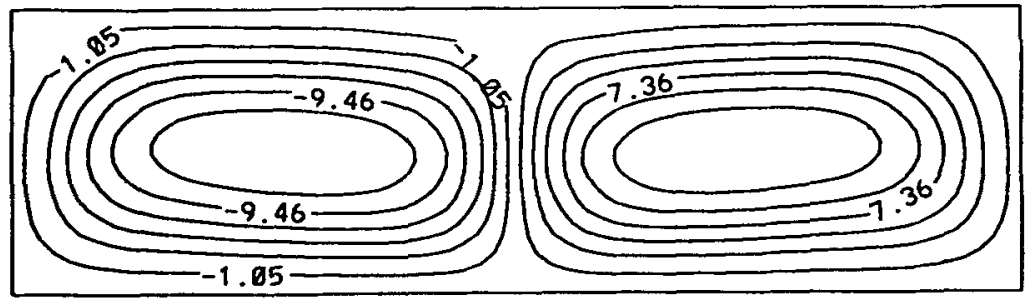

Figure 3. (a) Sectional streamlines, (b) velocity vectors, and (c) isotherms at vertical section $z=1 \cdot 7 ; \mathbf{R a}=20,000$ (Mukutmoni \& Yang 1993a).

Using suitable velocity perturbations, a two-roll flow pattern (figure 3) was developed. Based on a grid refinement study (Mukutmoni \& Yang 1993a), a $20 \times 20 \times 20$ grid was used for the computations. A third-order finite-difference scheme QUICK (Leonard 1983, pp. 211-26) was used. The finite-volume method and SIMPLEX algorithm (Van Doormal \& Raithby 1984) were implemented. Good quantitative agreement with experiments was found with respect to the frequencies and critical Rayleigh numbers. Qualitatively, the behaviour was identical. Both the computations and experiments showed a Hopf bifurcation followed by a period-doubling transition.

The numerical results showed that the oscillating temperature and velocity field exhibited a standing wave pattern propagating along the axis of the rolls. The nature of the oscillating temperature field is shown along a horizontal section in figure 4 as a series of contour plots spanning one complete oscillation cycle. Another study by Mukutmoni \& Yang (1992) revealed a similar standing wave pattern in a 3.3:1.9:1 box for a fluid Prandtl number of 0.5 (figure 5). These computations were based on the experiments of Maurer \& Libchaber (1979). Linear stability theory (Clever \& Busse 1974) predicts a travelling wave pattern for a horizontally unbounded domain. The standing wave observed in the simulations is an obvious extension of their results.

Several experiments of Rayleigh-Bénard convection in small enclosures (Gollub et al 1980, pp. 22-7; Libchaber \& Maurer 1981, pp. 259-86; Libchaber et al 1982) have shown that among the several bifurcation sequences observed there is one that approximates the classic period-doubling route to chaos observed in iterated maps 
(a)

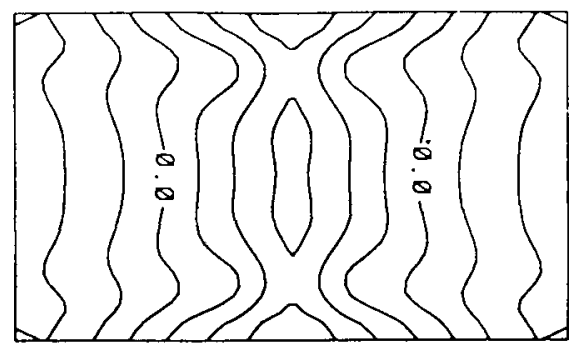

(c)

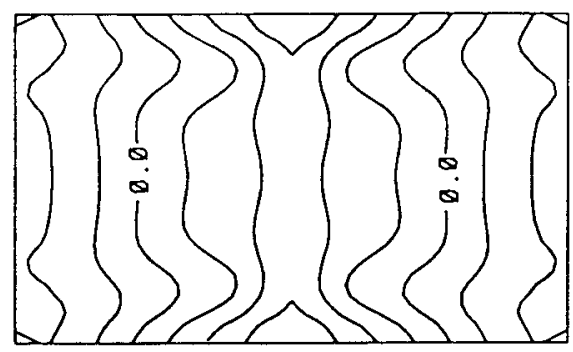

(e)

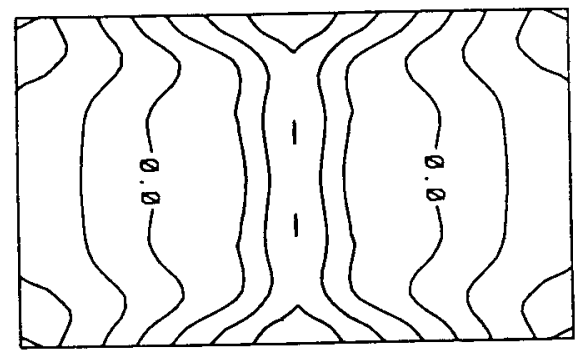

(g)

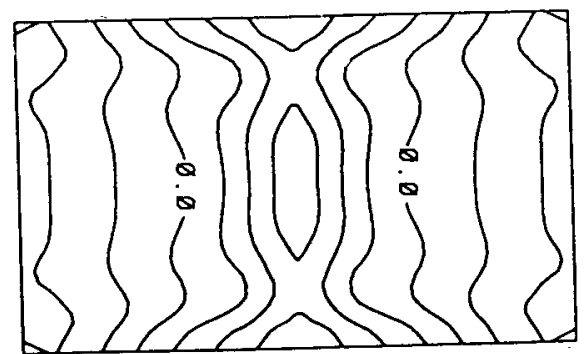

(b)

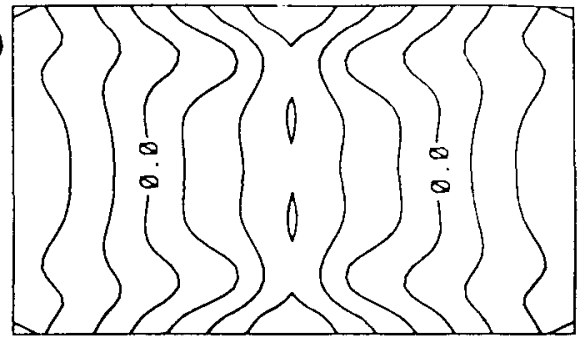

(d)

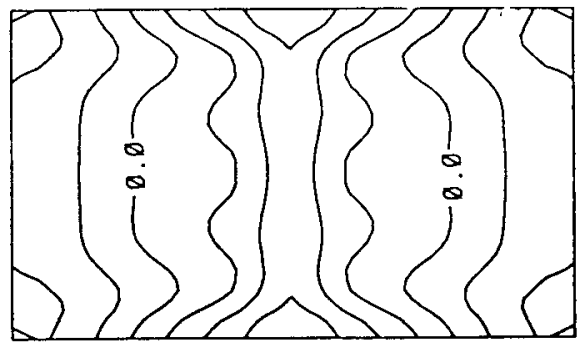

(f)

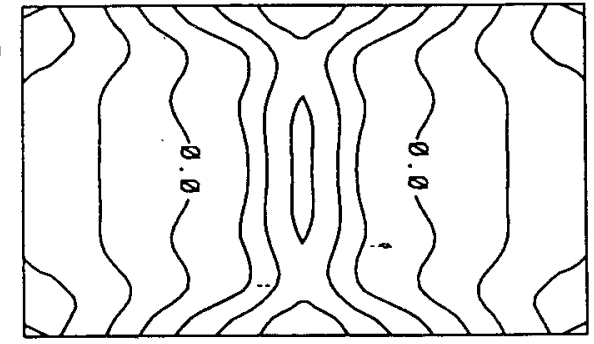

(h)

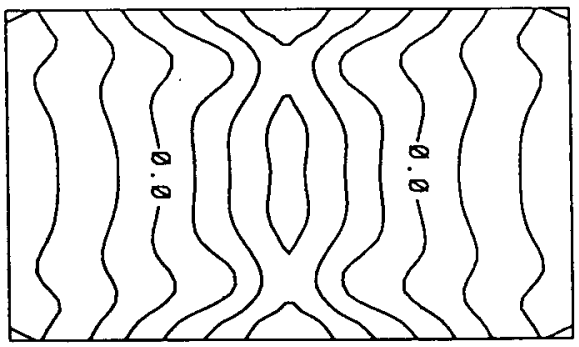

Figure 4. Instantaneous isotherms over a complete oscillation cycle; $\mathbf{R a}=35,000$ at the horizontal section $y=0.78$ (Mukutmoni \& Yang 1993a).

by Feigenbaum (1978). As an example, the following quadratic map exhibits such a behaviour as the control parameter $\lambda$ is increased,

$$
x_{n+1}=\lambda x_{n}\left(1-x_{n}\right)
$$

where $0 \leqslant x_{n} \leqslant 1$. In such a route to chaos (known as the Feigenbaum sequence) one observes an infinite cascade of period-doubling bifurcations. The intervals between successive critical control parameters get smaller and are in a constant ratio beyond a sufficiently larger number of bifurcations. The ratio is known as the Feigenbaum 
(a)

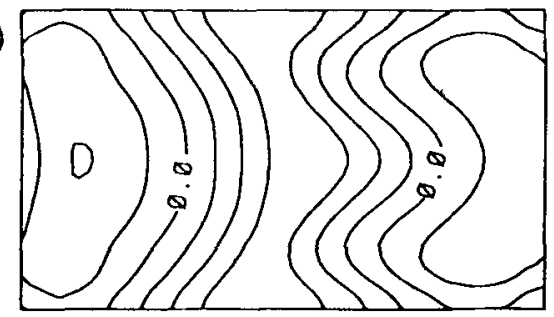

(c)

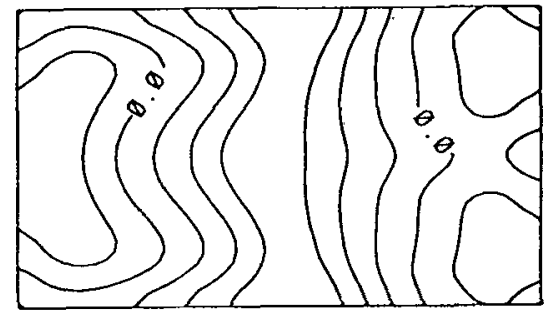

(e)

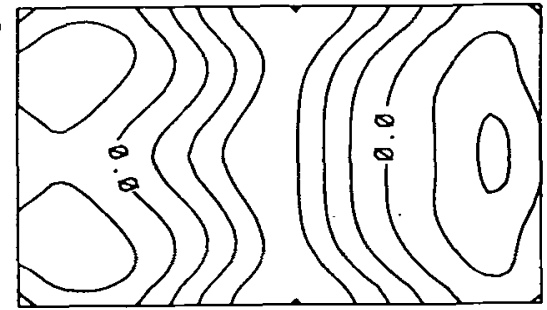

(g)

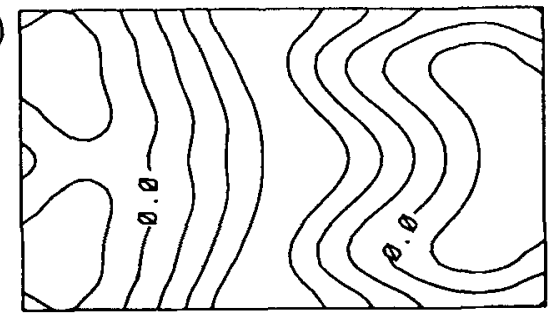

(b)

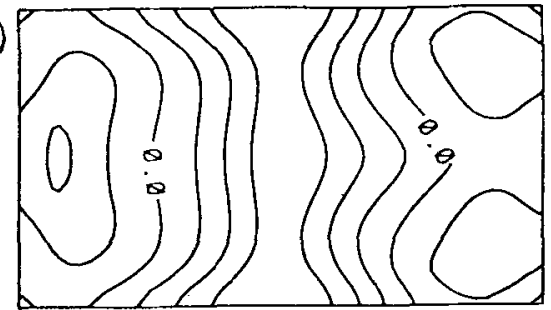

(d)

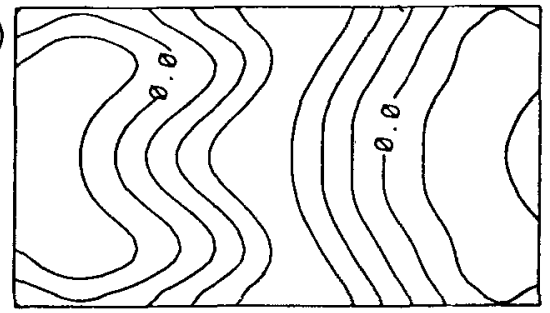

(f)

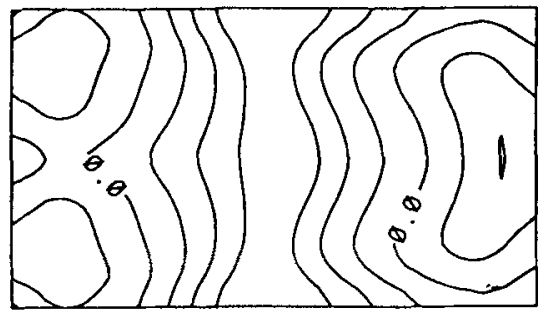

(h)

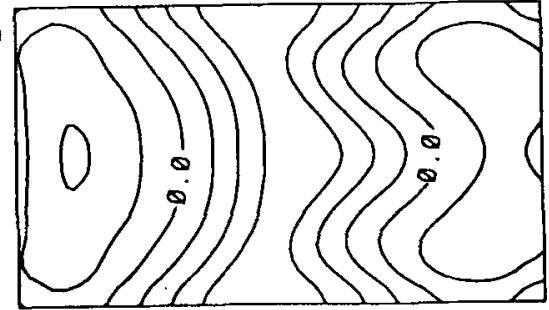

Figure 5. The oscillating isotherms over one complete period at the horizontal section $y=0.8$. Time interval is 0.023 (Mukutmoni \& Yang 1992)

number. Thus,

$$
\lim _{n \rightarrow \infty}\left(\lambda_{n}-\lambda_{n-1}\right) /\left(\lambda_{n+1}-\lambda_{n}\right)=4 \cdot 669201609 \ldots
$$

For the Rayleigh-Bénard system the sequence of sub-harmonic bifurcations experimentally observed is not an infinite cascade but stops at not more than five. This is followed by a bifurcation to quasi-periodicity and chaos. The reason why the Feigenbaum sequence is reproduced only partially for the RB system was looked into by us (Mukutmoni \& Yang 1993b). In our investigations (Mukutmoni 1991; Mukutmoni \& Yang 1993b), it was discovered that the bifurcation sequence strongly depended on the step increase in the Rayleigh number in the numerical study. For a step increase of 2000 , only one period-doubling bifurcation was observed (Mukutmoni \& Yang 
(a)

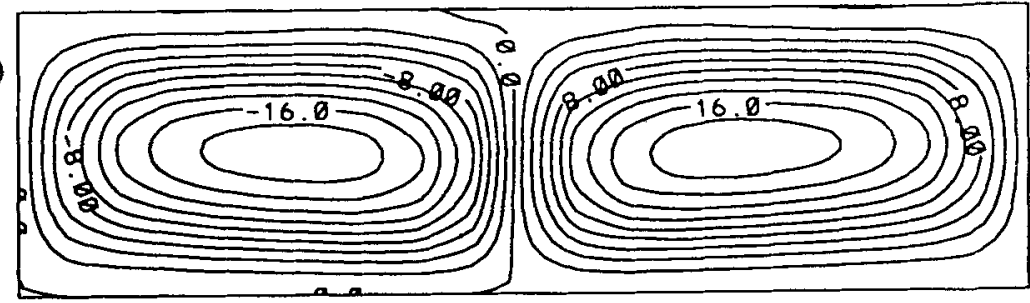

(b)

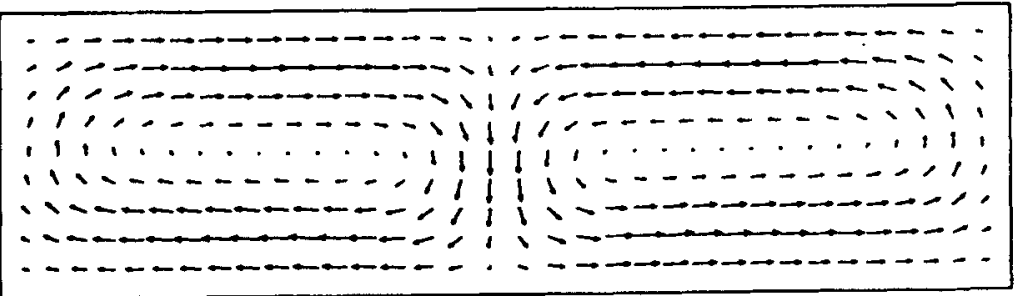

(c)

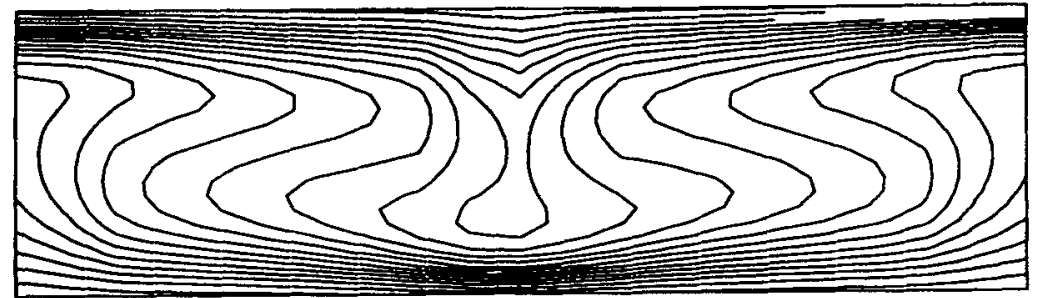

Figure 6. Mean velocity and temperature fields for $\mathrm{Ra}=37,000$ : (a) streamlines (b) velocity vectors, (c) isotherms (Mukutmoni \& Yang 1993b).
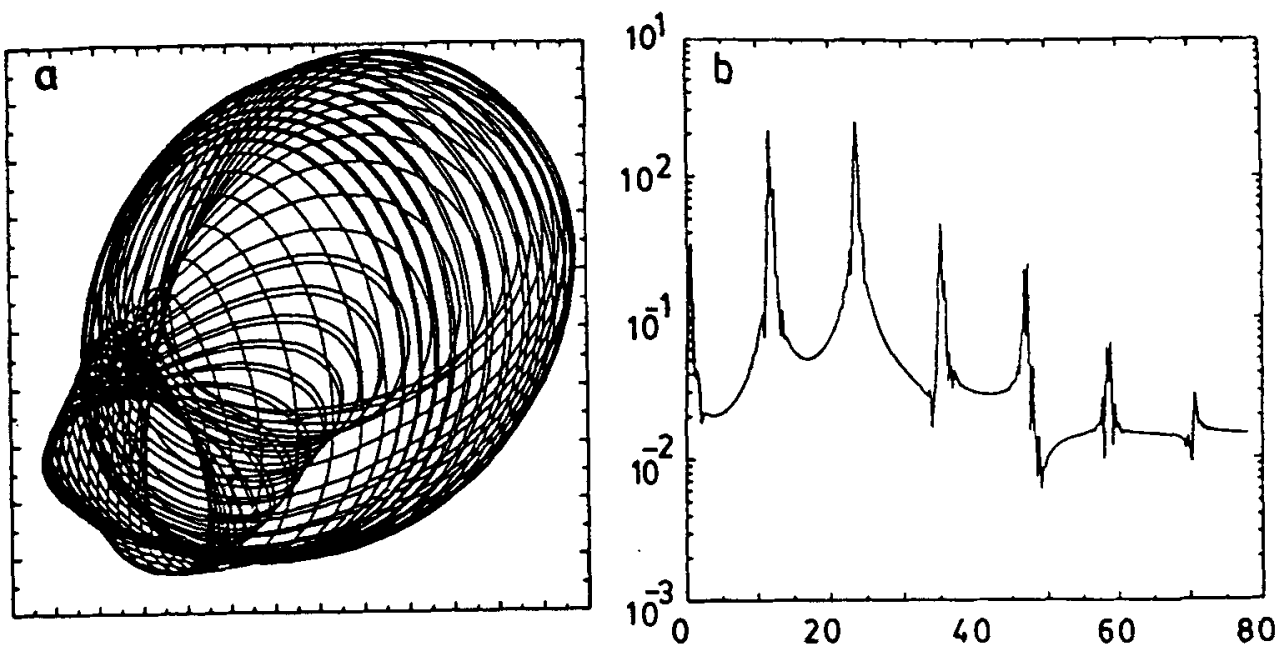

Figure 7. $\quad \mathrm{Ra}=39,000$; (a) phase trajectory, (b) spectral amplitudes (Mukutmoni \& Yang 1993b). 
1993b). For a smaller step increase, two subharmonic bifurcations were reported (Mukutmoni 1991).

After bifurcation to quasi-periodic flow, it was shown that the two-roll pattern developed asymmetry at $\mathrm{Ra}=\mathbf{3 7}, 000$ between the two rolls (figure 6). It was also reported that the independent frequency added to the system was an order of magnitude smaller than the first fundamental frequency (figure 7). The likely conclusion backed by experiments (Gollub et al 1980, pp. 22-7), is that as the Rayleigh number is increased, the symmetry between the rolls cannot be sustained and when that happens the Feigenbaum sequence is terminated. To further test this hypothesis, computation carried out by Mukutmoni \& Yang (1993b) artificially imposed such a symmetry. It was found that under such conditions, the Feigenbaum sequence is reproduced. One period-doubling sequence is shown in figure 8. Furthermore, the computations were able to predict the 'windows' of periodic flow between chaotic regimes as observed in the quadratic map (Parker \& Chua 1988).

It is rather surprising that a complicated system such as RB convection in small boxes for a certain parameter range can dynamically behave similar to one-dimensional nonlinear difference equations. However, as revealed in the experiments (Maurer \&
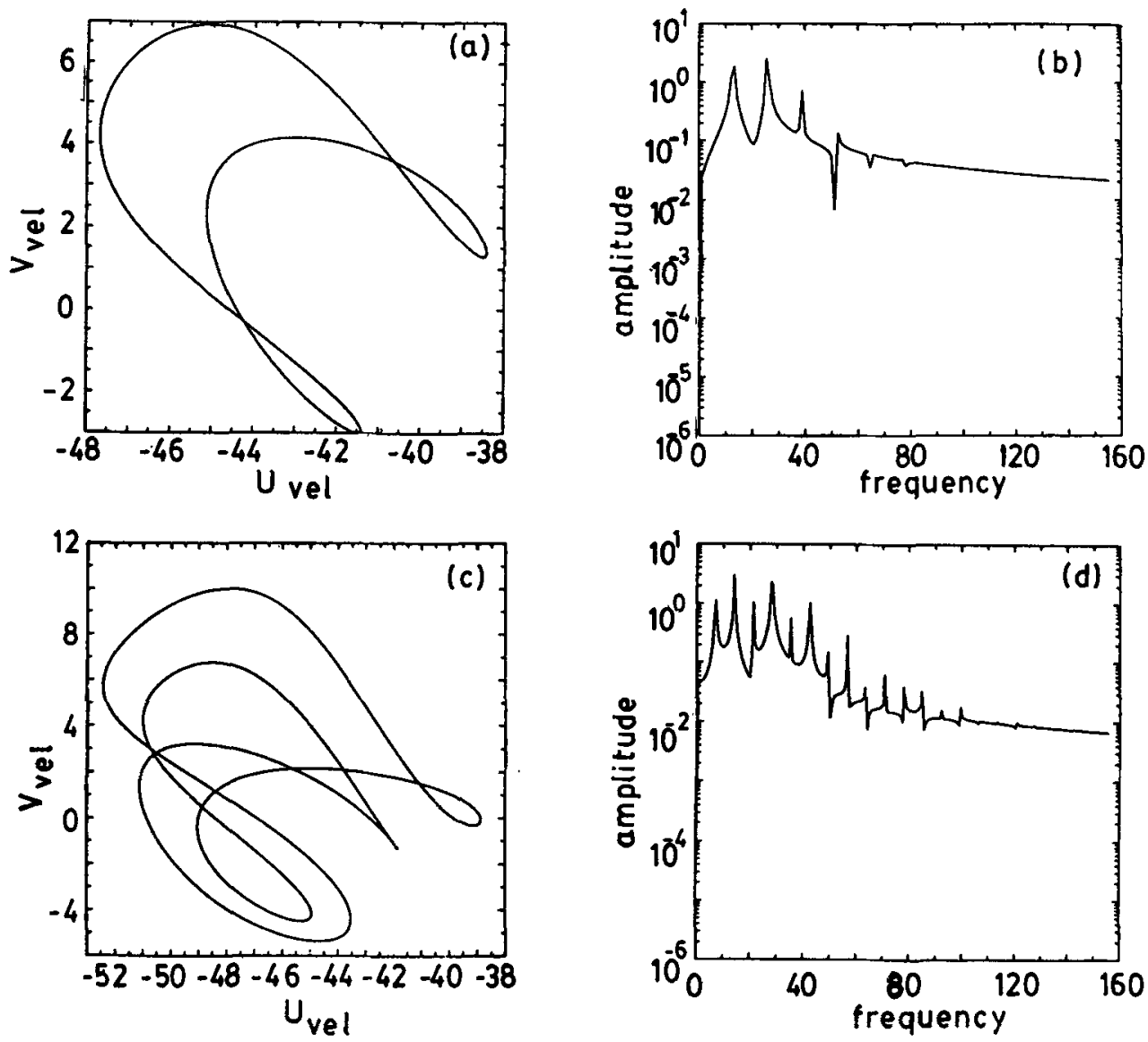

Figure 8. (a). Phase trajectory, (b) spectral amplitudes for $\mathrm{Ra}=47,000$; (c) phase trajectory, (d) spectral amplitudes for $R a=48,000$ (Mukutmoni \& Yang 1993b). 
Libchaber 1979; Gollub \& Benson 1980; Bergé et al 1982, pp. 123-48), the complete dynamical behaviour of the RB system is far richer than simple dynamical systems consisting of ordinary difference and differential equations. This is not surprising, since the equations of thermal convection are governed by a system of coupled nonlinear partial differential equations. It is also to be expected that the experiments have not revealed all the possible scenarios. With present computing resources, a thorough investigation of the dynamical behaviour is now tractable, and presents an exciting avenue for research to a fluid dynamicist and to a numerical analyst as a benchmark problem (Mukutmoni \& Yang 1991).

Mukutmoni \& Yang (1994a) further demonstrated the usefulness of numerical computations to gain specific physical insights when used in conjunction with experiments. Gollub \& Benson (1980) uncovered several routes to chaos and turbulence. In all cases, with one exception, the dynamical behaviour increased in temporal complexity as the Rayleigh number was increased. In one apparently anomalous case, for aspect ratios of 2.42 and 1.23 , and Prandtl number of 5.0, the flow reverted from a quasi-periodic to steady-state with an increase in Rayleigh number. Mukutmoni \& Yang (1944a) showed that the unexpected reversion to steadystate was caused by a bifurcation in the spatial pattern that stabilized the flow. Figure 9 shows the mean velocity and temperature field, before and after the bifurcation.

Preliminary numerical investigation into high Prandtl number fluids using the experiments of Arroyo \& Savirón (1992) was attempted by Mukutmoni et al (1993). Figure 10 shows the comparison with experiments in terms of pathlines. The experiments and numerical simulations were for an aspect ratio of 2.03 and 1.19 for silicone oil $(\operatorname{Pr}=130)$. One numerical difficulty associated with high Prandtl fluids is that time-dependence occurs at higher Rayleigh-numbers and that requires a finer grid and adequate resolution of the boundary layers.

(a)

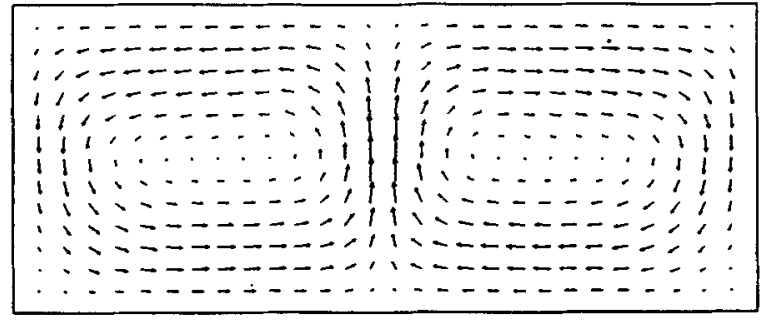

(c)

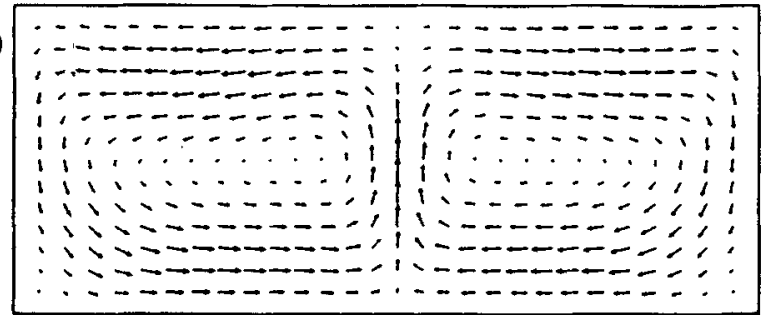

(b)

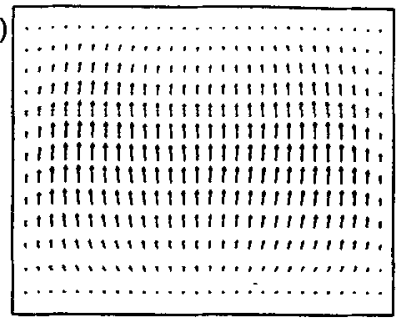

(d)

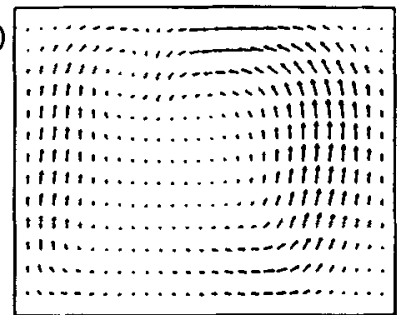

Figure 9. Velocity vectors in (a) $x-y$ plane for section $z=0.7$, and (b) $z-y$ plane for the section $x=1 \cdot 0, \mathrm{Ra}=40,000$, and in (c) $x-y$ plane for section $z=0.7$, and (d) $z-y$ plane for the section $x=1 \cdot 0, \mathrm{Ra}=130,000$ (Mukutmoni \& Yang 1994a). 

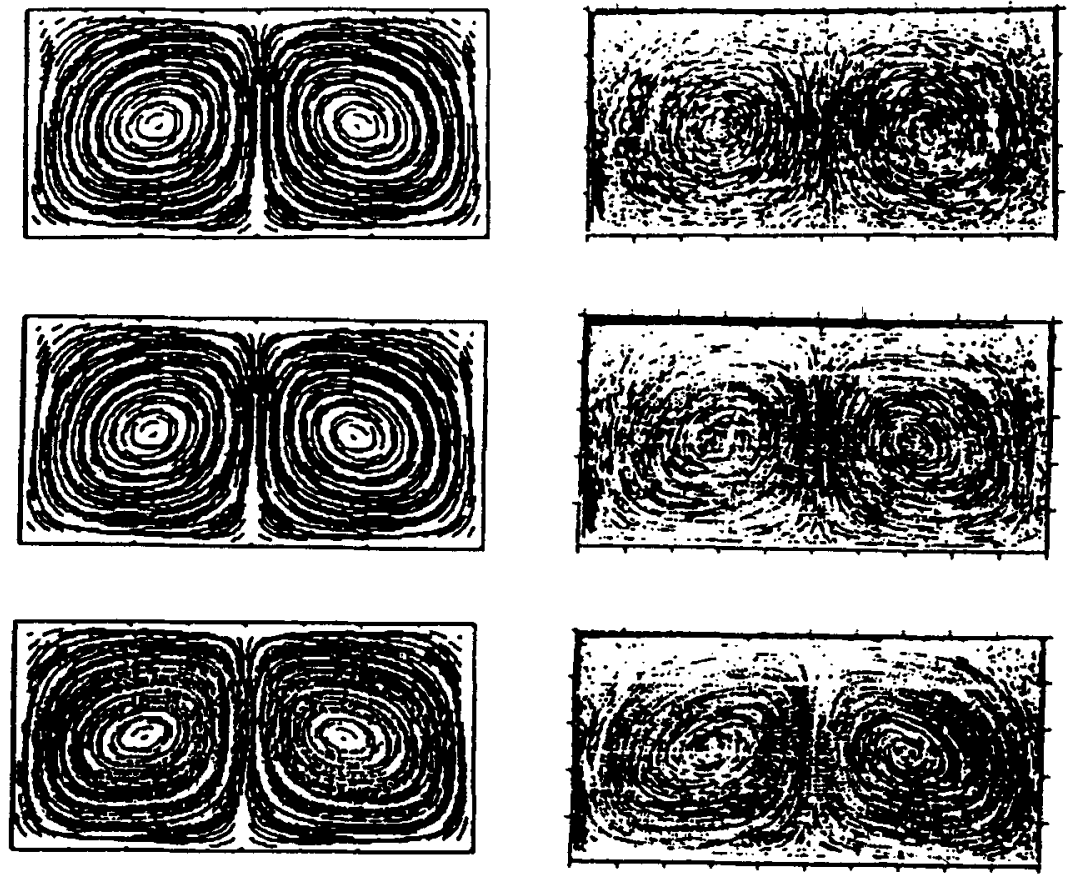

Figure 10. Computed pathlines (Mukutmoni et al 1993) on the left. Experimental results (Arroyo \& Savirón 1992) on the right. Rayleigh numbers starting from the top, 6319, 11101 and 22884 .

Thus far, the discussion has been mostly restricted to bifurcation in the temporal behaviour of the system. In the following section we discuss ongoing research in the study of the bifurcation in spatial patterns.

\section{Pattern selection in Rayleigh-Benard system}

It has long been known that the RB problem is degenerate, i.e., for the same set of governing parameters many solutions are possible (Busse 1978). This is a consequence of the nonlinearity of the problem. The mechanism on how different solutions to the problem evolve and compete and the process by which a particular flow configuration undergoes changes is broadly known as pattern selection. Some of the earliest work on the pattern selection problem was done by Busse and coworkers (Busse \& Whitehead 1974; Clever \& Busse 1974; Busse \& Clever 1979). In their works, they identified several instability mechanisms that influence the pattern selection process of RB convection in an infinite horizontally unbounded media. However, for an infinite case, the number of stable solutions is infinite. For roll convection, the stable solutions occur over a finite bandwidth of wavenumbers.

For large aspect ratio boxes (aspect ratios greater than 30 ) it has been experimentally shown (Busse 1978) that the instability mechanisms documented for the horizontally unbounded case in general apply, although the number of solutions is finite but large. For smaller enclosures, the evidence is insufficient. Experimental results indicate that 
at least some of the mechanisms do apply (Kolodner et al 1986; Kirchartz \& Oertel 1988). However, it is to be expected that new instability mechanisms would occur in the presence of lateral walls.

There have been several attempts to solve the problem with useful approximations of the governing equations. Using a perturbation expansion valid for regimes slightly above the critical, Newell \& Whitehead (1969) and Segel (1969) derived an amplitude equation. Subsequent researchers have improved and generalized this amplitude equation to study growth and saturation of the rolls. Using this approach, Greenside \& Coughran (1984) predicted (experimentally confirmed by Gollub \& Heutmaker 1984) that rolls tend to intersect rigid non-slip walls in an approximately perpendicular direction. The amplitude equation is a two-dimensional nonlinear partial differential equation that approximates (1) to (5). It has the following form,

$$
\partial \phi / \partial t=\left[\varepsilon-\left(\nabla^{2}+1\right)^{2}\right] \phi-\phi^{3},
$$

where $\varepsilon$ is the perturbation parameters $\left(\mathrm{Ra} / \mathrm{Ra}_{c}\right)-1$. The dependent variable $\phi$ is the amplitude.

Greenside \& Coughran (1984) studied the pattern selection problem by numerically solving the amplitude equation and imposing random initial conditions and integrating in time. The results indicate that irregular patterns or defects do develop but they smoothen out for large Rayleigh numbers. The stationary solutions were found to be symmetric. It was determined that the time scales needed to achieve a stationary solution was proportional to the horizontal diffusion scale.

Nevertheless, the amplitude equations are valid only slightly above the critical Rayleigh number. To really model the phenomena accurately in the high Rayleigh number regime, the full Boussinesq equation must be solved. The problem is less tractable than a small box since a large computational grid is required. Furthermore, since the relaxation time is proportional at least to the horizontal diffusion time, the simulations must be carried out for a longer time.

RB convection in an intermediate aspect ratio box was experimentally investigated by Kolodner et al (1986). They concluded that the observations were consistent with the results of two-dimensional stability theory, provided one makes plausible allowances for the experimental imposition of finite lateral boundaries. The focus of most experimentalists has been on roll convection. One well-documented phenomenon experimentally observed in small and intermediate boxes is the decrease in the number of rolls with an increase in Rayleigh number (Kolodner et al 1986; Leith 1987; Kirchartz \& Oertel 1988). According to linear stability theory, the loss of roll phenomena, as it is sometimes referred to, is an outcome of the skewed-varicose instability (Busse 1978).

The loss of rolls was numerically investigated by Mukutmoni \& Yang (1992) for a small box $(4: 2: 1)$ for a Prandtl number of 0.71 . The transition from 4 to 3 rolls was documented as shown in figure 11 . The transition sequence shows the typical slanting of the rolls as well as the thinning and thickening of the distorted rolls in the time sequence. In our computations (Mukutmoni \& Yang 1994b), using a $98 \times 20 \times 50$ grid, we simulated a transition from 10 to 8 rolls, for an enclosure of aspect ratios 10.61 and 5.32 and Prandtl number of 3.5. The transition sequence is shown in figure 12. Our results (Mukutmoni \& Yang 1994) also showed that there is a generation of vertical vorticity or swirl in the transition process. This means that 
(a)
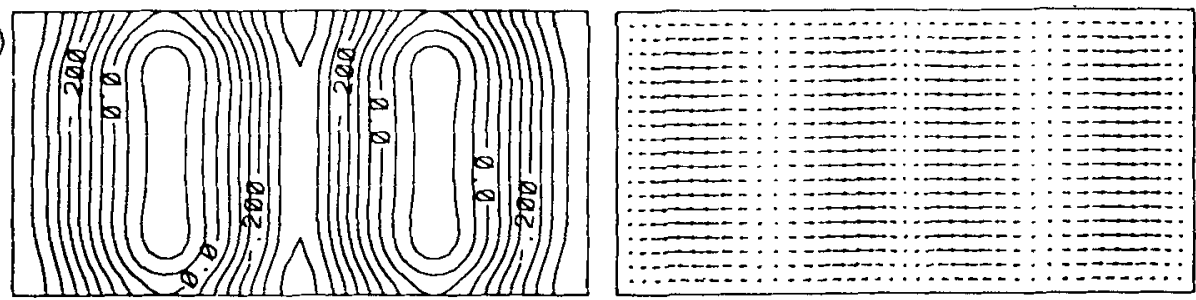

(b)
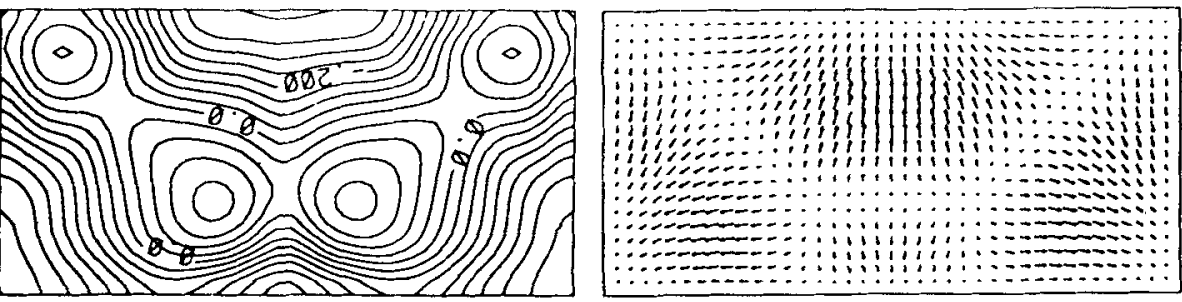

(c)
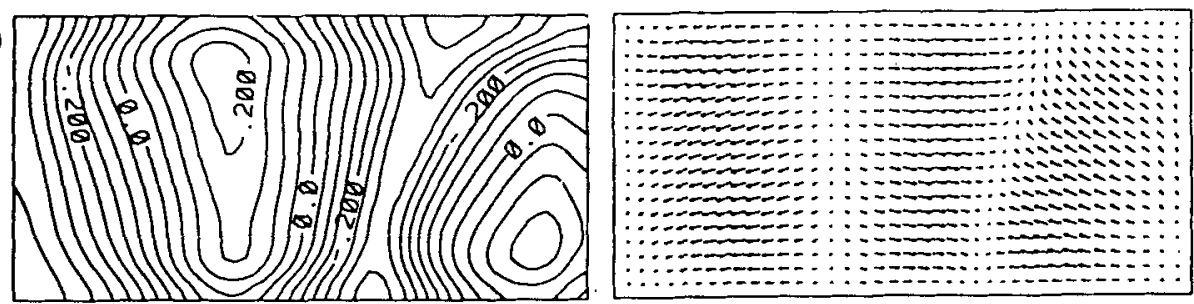

(d)
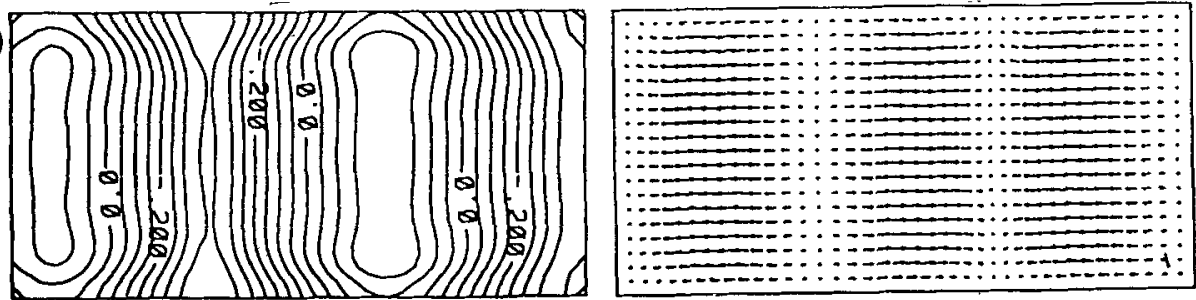

Figure 11. Transition sequence from 4 to 3 cells. $R a=10,000$; horizontal section $y=0.8$ (Mukutmoni \& Yang 1992).

the transition process is not governed entirely by the skewed-varicose instability for which there is no vertical vorticity (Busse 1978). The numerical computations were an accurate simulation of the experiments of Kolodner et al (1986).

Another related issue of roll convection is related to the alignment of the rolls. Early theoretical (Davis 1967) and experimental results (Stork \& Müller 1972) seemed to suggest that only rolls parallel to the short side of a rectangular container are stable. However, we (Mukutmoni \& Yang 1992) showed that rolls parallel to the long side for a small enclosure of aspect ratio 3.5 and 2.1 and Prandtl number 2.5 are stable below a certain critical Rayleigh number. The conclusion of the study (Mukutmoni \& Yang 1992) was that long rolls are metastable (unstable to finite perturbations) and definitely less stable than rolls parallel to the short side. Experiments of Kolodner et al (1986) found stable rolls parallel to the long side, for intermediate aspect ratio boxes.

Pattern selection and flow transitions in intermediate aspect ratio boxes are much more complicated than smaller boxes due to the less restrictive influence of the 
(a)

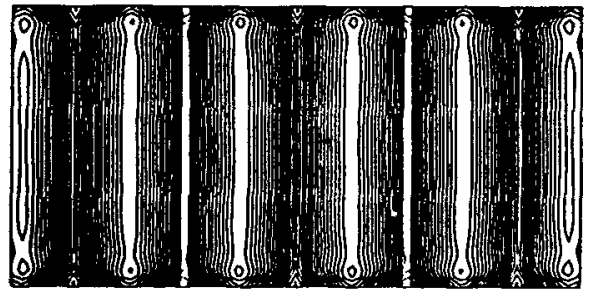

(c)

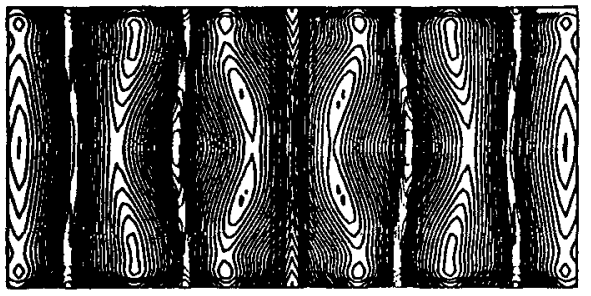

(b)

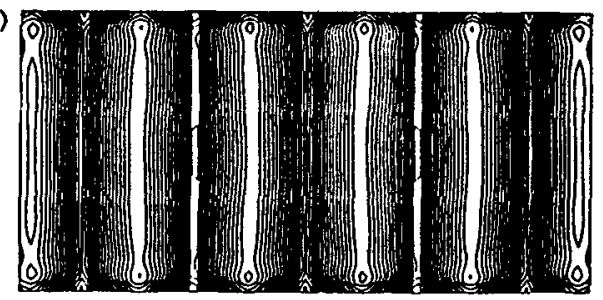

(d)

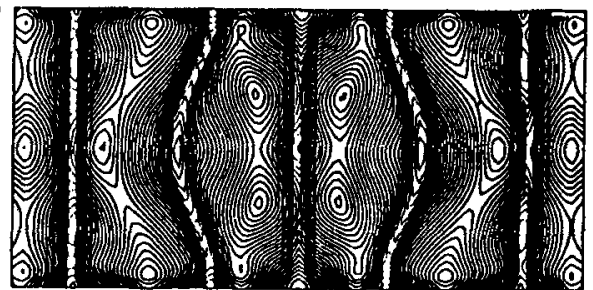

(e)
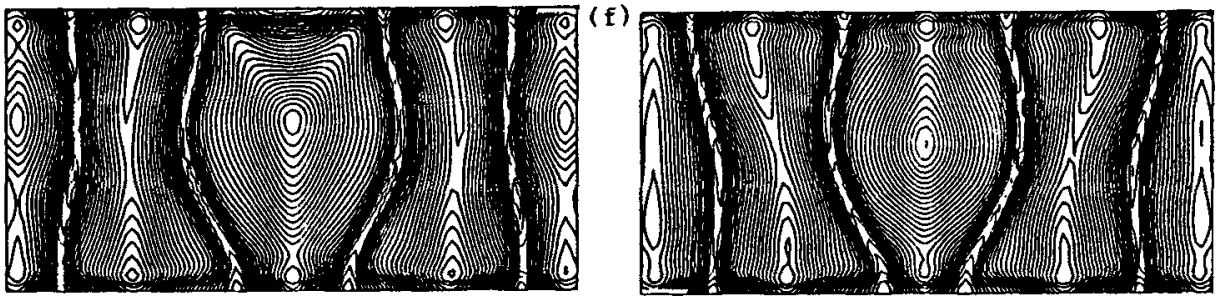

(8)
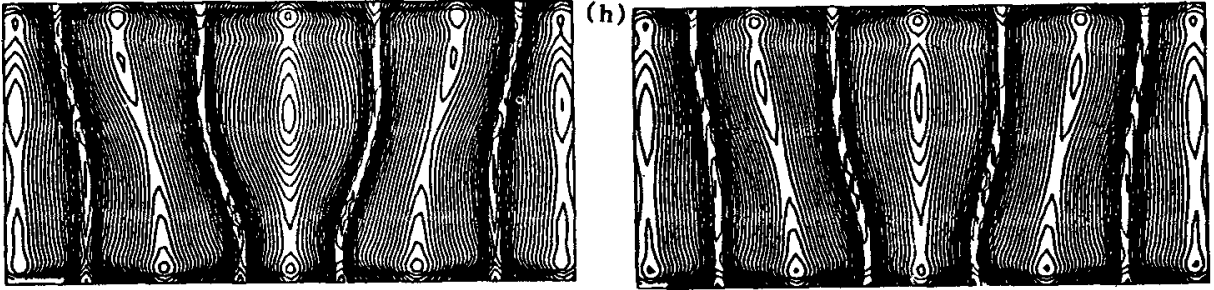

(i)
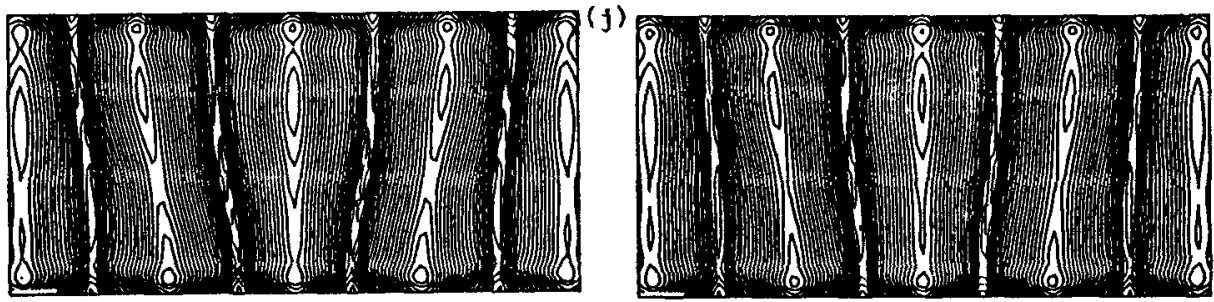

(k)
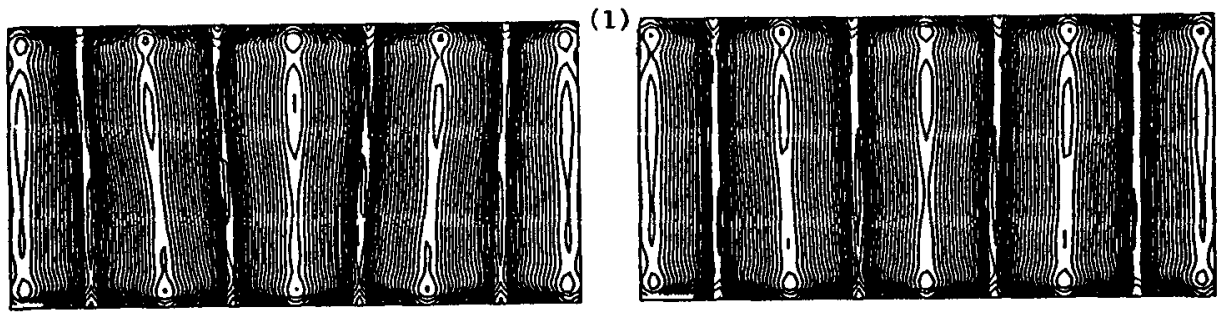

Figure 12. Pattern transition at $\mathrm{Ra}=17,000$ represented in terms of isotherms at horizontal section $y=0 \cdot 7$. Time interval of $t=1$ (Mukutmoni \& Yang 1994b). 
(a)

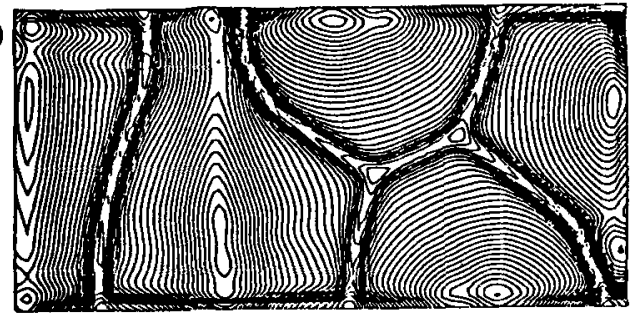

(c)

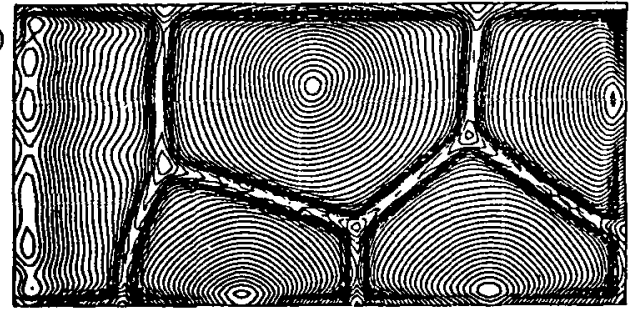

(e)

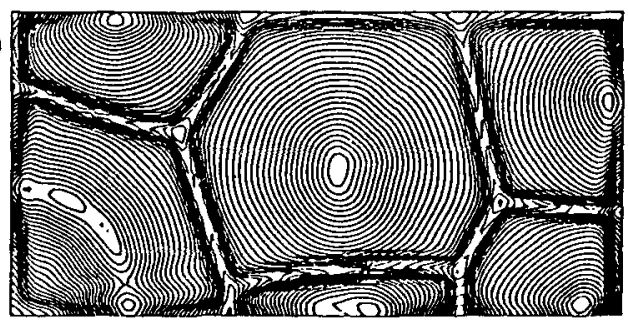

(b)

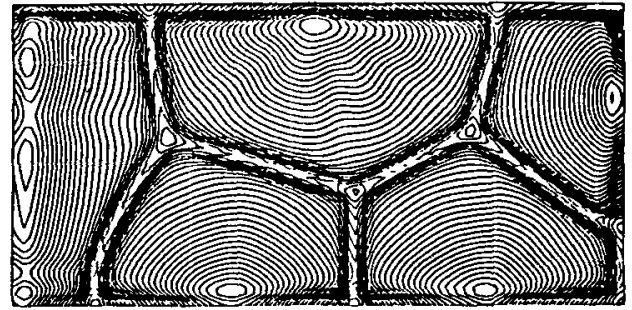

(d)

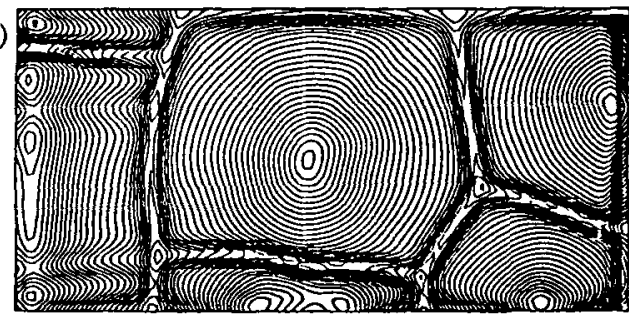

Figure 13. Non-roll pattern transition at $\mathrm{Ra}=24,000$ (Mukutmoni \& Yang 1994b).

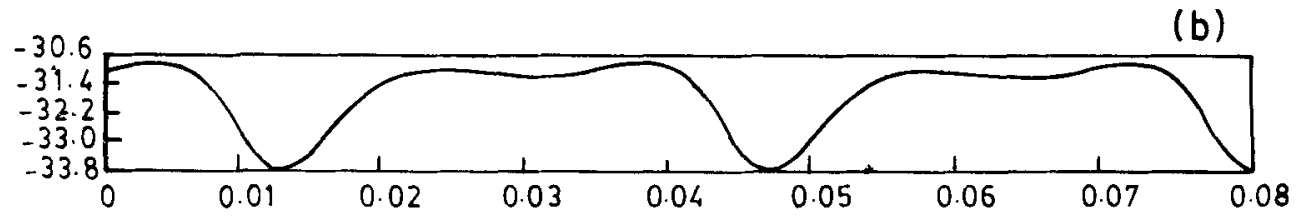

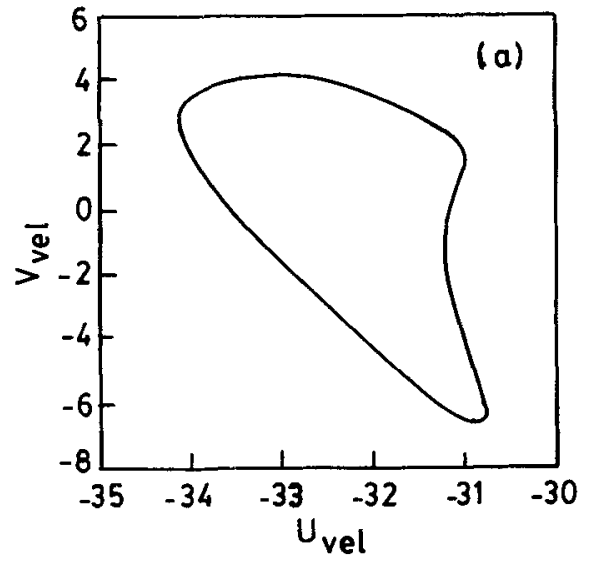

Figure 14. (a) Phase trajectory in the $u-v$ plane, and (b) $u$-velocity as a function of time, for $R a=24,000$ (Mukutmoni \& Yang 1994b). 
sidewalls. Some experiments hint at a much more complicated temporal behaviour (Walden et al 1984) at higher Rayleigh numbers than small enclosures. The flow patterns are more complicated. Several non-roll patterns have been documented. For moderately high Prandtl fluids, bimodal convection has been documented experimentally (Busse 1978). Another interesting non-roll pattern is the "spoke-pattern" convection (Kolodner et al 1986) which has a highly complicated three-dimensional structure and is time-dependent as well. We (Mukutmoni \& Yang 1994b) documented steady oscillatory convection with a polygonal planform. The planform is shown in figure 13 and the dynamical behaviour is shown in figure 14. Such a pattern has however not been observed in experiments.

\section{Research needs and concluding remarks}

From our discussion it appears that numerical simulation of RB convection in enclosures is a very worthwhile and rewarding research area. However, it is clear that the numerical studies have yet to catch up with the range and scope of the experiments. The review shows that successful simulations have been performed for small aspect ratio enclosures up to the chaotic regime for some cases. Nevertheless, simulations have not covered the entire range of dynamical behaviour. Although experiments are available, there has been no study investigating the intermittency and phase-locking behaviour that has been observed. Systematic and extensive studies of high-Prandtl fluids in enclosures are absent thus far.

It is also worthwhile to look at convection in small enclosures for non-rectangular geometries such as with cylindrical lateral walls or polygonal domains. There has not been any study as yet. Even for rectangular domains, turbulent convection in enclosures at the lower Rayleigh number and (sometimes referred to as "soft" turbulence) has not received the attention it deserves. All numerical studies on turbulent thermal convection use some degree of approximation such as periodicity in the horizontal directions or slip horizontal walls such as the works of Sirovich \& Park (1990). One obvious problem for RB onvection for high Rayleigh numbers in the turbulent regime is the tractability. However, with the greater availability of powerful workstations and massively parallel machines these problems would be feasible, and lead to a greater understanding of the physics.

For intermediate aspect ratio enclosures, the computational problem is more difficult. Not surprisingly, there have been extremely few studies that tackle the problem in all its complexity. However, due to the more complex physics associated with the problem, it is a more rewarding area. The phenomena of RB convection in intermediate boxes are not well known. The first challenge would be to take a closer look at the experimentally observed flow patterns. In particular, the non-roll patterns have not been looked into at all.

Thermal convection at high Rayleigh numbers is a computationally difficult numerical problem. In this article, we have described, in certain detail, some of the computational techniques used in the various studies. We make no specific recommendations on the type of numerical technique employed. However, higher order methods are to be preferred in order to minimize numerical diffusion. The class of numerical techniques used by researchers include spectral, finite element and finite volume methods. For a complete exposition on the spectral methods the reader is referred to the monograph by Canuto et al (1988). An excellent reference for the finite volume method is the book by Fletcher (1988). 
Higher order finite difference and finite volume methods have only recently been applied to numerical problems. As an example Lele (1992) describes the compact differencing schemes. Rai \& Moin (1991) illustrate higher order upwind-weighted schemes for simulating turbulent flow. The issue of the required mesh density is highly problem dependent. For the RB problem, as described earlier, it depends strongly on the Rayleigh number and the Prandtl numbers. Experimental results are needed to primarily validate the grid resolution.

\section{List of symbols}

$A_{x}, A_{z} \quad L_{x} / L$ and $L_{z} / L$, aspect ratios in the $x$ and $z$ directions respectively;

$g \quad$ acceleration due to gravity, $\mathrm{m} / \mathrm{s}^{2}$;

$L \quad$ height of the enclosure, $\mathrm{m}$;

$L_{x}, L_{z} \quad$ dimensions of enclosure in $x$ and $z$ directions $(\mathrm{m})$ respectively;

Pr Prandtl number;

Ra Rayleigh number;

$\mathrm{Ra}_{c} \quad$ critical Rayleigh number;

$t \quad$ non-dimensional time;

$T$ non-dimensional temperature;

$\Delta T \quad$ temperature difference, $\mathrm{K}$;

$\mathbf{U}$ non-dimensional velocity vector;

$u, v, w \quad$ non-dimensional $x$-, $y$ - and $z$-direction velocites respectively;

$x, y$ non-dimensional horizontal and vertical spatial coordinates respectively;

$z \quad$ non-dimensional spatial coordinate in the direction of depth;

$\alpha \quad$ thermal diffusivity, $\mathrm{m}^{2} / \mathrm{s}$;

$\beta \quad$ coefficient of volume expansion, $1 / \mathrm{K}$;

$\varepsilon \quad$ perturbation parameter;

$\lambda$ bifurcation parameter;

$v \quad$ kinematic viscosity, $\mathrm{m}^{2} / \mathrm{s}$;

$\rho \quad$ density, $\mathrm{kg} / \mathrm{m}^{3}$;

$\phi \quad$ non-dimensional amplitude of perturbation.

\section{References}

Ahlers G, Behringer R P 1978 The Rayleigh-Bénard instability and the evolution of turbulence. Suppl. Prog. Theor. Phys. 64: 186-201

Arroyo M P, Savirón J M 1992 Rayleigh-Bénard convection in a small box: Spatial features and thermal dependence of the velocity field. J. Fluid Mech. 235: 325-348

Bénard H 1900 Les Tourbillons Cellulaires Dans Une Nappe Liquide. Revue Générale des Sciences Pures et Appliquées 11: 1261-1271 and 11: 1309-1328

Bergé P, Dubois M, Croquette V 1982 Approach to Rayleigh-Bénard turbulent convection in different geometries. Convective transport and instability phenomenon (eds) $\mathrm{J}$ Zierep, H Oertel Jr (Karlsruhe: G Braun)

Bühler K, Kirchartz K R, Oertel H Jr 1979 Steady convection in a horizontal fluid layer. Acta Mech. 31: 155-171

Busse F H 1978 Nonlinear properties of thermal convection. Rep. Prog. Phys. 41: 1929-1967

Busse F H, Clever R M 1979 Instability of convection rolls in a fluid of moderate Prandtl number. J. Fluid Mech. 91: 319-335

Busse F H, Whitehead J A 1974 Oscillatory and collective instabilities in large Prandtl number convection. J. Fluid Mech. 66: 67-79 
Canuto C, Hussaini M Y, Quateroni A, Zang T A 1988 Spectral methods in fluid dynamics (New York: Springer-Verlag)

Catton I 1970 Convection in a closed rectangular region: The onset of motion. ASME J. Heat Transfer 92: 186-187

Chandrasekhar S 1961 Hydrodynamic and hydromagnetic stability (Oxford: University Press)

Charleson G S, Sani R L 1970 Thermo-convective instability in a bounded cylindrical fluid layer. Int. J. Heat Mass Transfer 13: 1479-1496

Clever R M, Busse F H 1974 Transition to time dependent convection. J. Fluid Mech. 65: 625-645

Clever R M, Busse F H 1987 Nonlinear oscillatory convection. J. Fluid Mech. 176: 403-417

Davis S H 1967 Convection in a box: Linear theory. J. Fluid Mech. 30: 465-478

Feigenbaum M J 1978 Quantitative universality for a class of nonlinear transformations. $J$. Stat. Phys. 19: 25-52

Fletcher C A J 1988 Computational techniques for fluid dynamics (New York: Springer-Verlag)

Gollub J P, Benson S V 1980 Many routes to turbulent convection. J. Fluid Mech. 100: 449-470

Gollub J P, Benson S V, Steinman J F 1980 A subharmonic route to turbulent convection. Nonlinear dynamics (ed.) H G Helleman (New York: Academy of Sciences)

Gollub J P, Heutmaker M S 1984 Turbulence and chaotic phenomena in fluids (ed.) T Tatsumi (Amsterdam: North-Holland)

Greenside H S, Coughran W M Jr 1984 Nonlinear pattern formation near the onset of Rayleigh-Bénard convection. Phys. Rev. A30: 398-428

Kessler R 1987 Nonlinear transition in three-dimensional convection. J. Fluid Mech. 174: 357-379

Kirchartz K R, Oertel H Jr 1988 Three-dimensional thermal cellular convection in rectangular boxes. J. Fluid Mech. 192: 249-286

Kolodner P, Walden R W, Passner A, Surko C M 1986 Rayleigh-Bénard convection in an intermediate-aspect-ratio rectangular container. J. Fluid Mech. 163: 195-226

Leith J R 1987 Successive transitions of steady states in moderate size containers of air heated from below and cooled above. Bifurcation phenomena in thermal processes and convection HTD (New York: Am. Soc. Mech. Eng.) vol. 94

Lele S K 1992 Compact finite difference schemes with spectral-like resolution. J. Comput. Phys. 103: $16-42$

Leonard B P 1983 A convectively stable, third-order accurate finite-difference method for steady two-dimensional flow and heat transfer. Numerical properties and methodologies in heat transfer (ed.) T M Shih (Washington, DC: Hemisphere)

Libchaber A, Larouche C, Fauve L 1982 Period-doubling cascade in mercury, a quantitative measurement. J. Phys. Lett. 43: L211-L216

Libchaber A, Maurer J 1981 A Rayleigh-Bénard experiment: Helium in a small box. Nonlinear phenomena at phase transitions and instabilities (ed.) T Riste (New York: Plenum)

Maurer J, Libchaber A 1979 Rayleigh-Bénard experiment in liquid helium: Frequency locking and the onset of turbulence. J. Phys. Lett. 40: L419-L423

Mukutmoni D 1991 Transitions and bifurcations in Rayleigh-Bénard convection in a small aspect ratio. $\mathrm{Ph} \mathrm{D}$ dissertation, Department of Aerospace and Mechanical Engineering, University of Notre Dame, Notre Dame, IN

Mukutmoni D, Kelleher M D, Joshi Y K 1993 AMPHIB: A users manual. Technical Report NPS-ME-93-001, Naval Postgraduate School, Monterey, CA

Mukutmoni D, Yang K T 1991 Transition to oscillatory flow in Rayleigh-Bénard convection in a three-dimensional box. ASME Paper No. 91-HT-10

Mukutmoni D, Yang K T 1992 Wavenumber selection for Rayleigh-Bénard convection in a small aspect ratio box. Int. J. Heat Mass Transfer 35: 2145-2159

Mukutmóni D, Yang K T 1993a Rayleigh-Bénard convection in a small aspect ratio enclosure: Part I-Bifurcation to oscillatory convection. J. Heat Transfer 115: 360-366

Mukutmoni D, Yang K T 1993b Rayleigh-Bénard convection in a small aspect ratio enclosure: Part Il-Bifurcation to chaos. J. Heat Transfer 115: 367-376

Mukutmoni D, Yang K T 1994a Thermal convection in small enclosures: An atypical bifurcation sequence. Int. J. Heat Mass Transfer (accepted for publication)

Mukutmoni D, Yang K T 1994b Pattern selection for Rayleigh-Bénard convection in intermediate aspect ratio boxes. Numer. Heat Transfer (Accepted for publication) 
Newell A C, Whitehead J A 1969 Finite bandwidth, finite amplitude convection. J. Fluid Mech. 38: 279-303

Oertel H Jr 1982 Thermal instabilities. Convective transport and instability phenomena (eds) J Zierep, H Oertel Jr (Karlsruhe: G Braun)

Parker T S, Chua L O 1988 Practical numerical algorithms for chaotic systems (New York: Springer-Verlag)

Rai M M, Moin P 1991 Direct simulations of turbulent flow using finite-difference schemes. J. Comput. Phys. 96: 15-53

Rayleigh L 1916 Convective currents in a horizontal layer of fluid when the higher temperature is on the under side. Philos. Mag. 32: 529-575

Ruelle D, Takens F 1971 On the nature of turbulence. Commun. Math. Phys. 20: 167-192

Segel L A 1969 Distant side-walls cause slow amplitude modulation of cellular convection. J. Fluid Mech. 38: 203-224

Sirovich L, Park H 1990 Turbulent thermal convection in a finite domain. Phys. Fluids A9: $1649-1668$

Stork K, Müller U 1972 Convection in boxes: Experiments. J. Fluid Mech. 54: 599-611

Upson C D, Gresho P M, Sani R L, Chan S T, Lee R L 1981 A thermal convection simulation in three dimensions by a modified finite element method. Numerical properties and methodologies in heat transfer (ed.) T M Shih (Washington, DC: Hemisphere)

Urata K 1986 Low dimensional chaos in Boussinesq convection. Fluid Dynamics Res. 1: 257-282

Van Doormal J P, Raithby G D 1984 Enhancements of the simple method for predicting incompressible fluid flows. Numer. Heat Transfer 17: 147-163

Walden R W, Kolodner P, Passner A, Surko C M 1984 Non-chaotic Rayleigh-Bénard convection with four and five incommensurate frequencies. Phys. Rev. Lett. 53: 242-245

Yahata H 1984 Onset of chaos in the Rayleigh-Bénard convection. Prog. Theor. Phys. Suppl. 79: $25-74$

Yang K T 1988 Transitions and bifurcations in laminar buoyant flows in confined enclosures. J. Heat Transfer 110: 1191-1204

Yang K T, Mukutmoni D 1992 Computational aspects of studies of buoyant enclosure flows. Computers and computing in heat transfer science and engineering (eds) W Nakayama, K T Yang (Boca Raton, FL: Begell House-CrC Press) 